\title{
VEGETATIVE FOREIGN BODY OF NECK: A RARE EXPERIENCE
}

\section{Raman Choudhary ${ }^{1}$, H. S. Bhuie' ${ }^{2}$, Navneet P. Mathur ${ }^{3}$, Arjun Nath Yogi ${ }^{4}$, Hitesh Verma ${ }^{5}$}

\section{HOW TO CITE THIS ARTICLE:}

Raman Choudhary, H. S. Bhuie, Navneet P. Mathur, Arjun Nath Yogi, Hitesh Verma. "Vegetative Foreign Body of Neck: A Rare Experience". Journal of Evolution of Medical and Dental Sciences 2014; Vol. 3, Issue 21, May 26; Page: 5781-5784, DOI: $10.14260 /$ jemds/2014/2664

ABSTRACT: Foreign bodies are a common problem seen in otolaryngological practice ${ }^{1}$. Four year male patient presented with history of accidental ingestion of wooden foreign body in oral cavity. On examination, irregular wound was present in right anterior tonsillar pillar. The neck showed hard mass deep to upper third of sternocleidomastoid muscle. The contrast enhancing CT scan showed hypo intense mass in right carotid triangle between internal carotid artery and internal jugular vein. We are presenting here our rare experience

KEYWORDS: Vegetative foreign body, Carotid artery, internal jugular vein.

INTRODUCTION: Foreign bodies are a common problem seen in otolaryngological practice ${ }^{1}$. Of the reported foreign bodies, wooden foreign bodies are a rare entity. The penetrating foreign body in the neck has a special apprehension because of the constellations of vital structures in the neck. ${ }^{2}$ They may cause life-threatening suppurative or vascular complication. The wooden foreign body creates more diagnostic difficulties. ${ }^{3}$ It may initially disappear in the computerized tomography (CT) scan.

The management could be difficult due to its easy fragility and infectious nature. The early wound exploration and proper wound debridement with proper antimicrobial coverage are the prime factors in curtailing morbidity and mortality. ${ }^{3}$

CASE REPORT: Four year male patient presented with history of accidental ingestion of wooden stick in oral cavity and it was also pushed the skin outward in upper part of neck. The relatives of the patient were made a futile attempt to remove the wooden piece and left part of it. He also had history of bleeding from oral cavity which was small in amount, stop by there own.

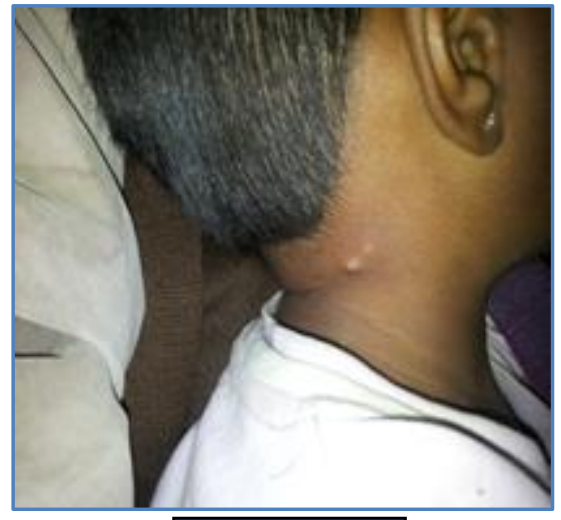

Picture 1

There was no history of loss of consciousness, excessive bleeding, difficulty in breathing and swallowing. The general physical examination was normal with bilateral symmetrical carotid and superficial temporal pulsation. The examination of oral cavity showed irregular $1-2 \mathrm{~cm}$ wound in 


\section{CASE REPORT}

right anterior tonsillar pillar. The neck showed bony hard mass deep to upper third of sternocleidomastoid muscle (picture 1).

The contrast-enhanced CT (CECT) scan of head and neck region revealed hypointense area in right carotid triangle with splaying of internal carotid artery and internal jugular vein.
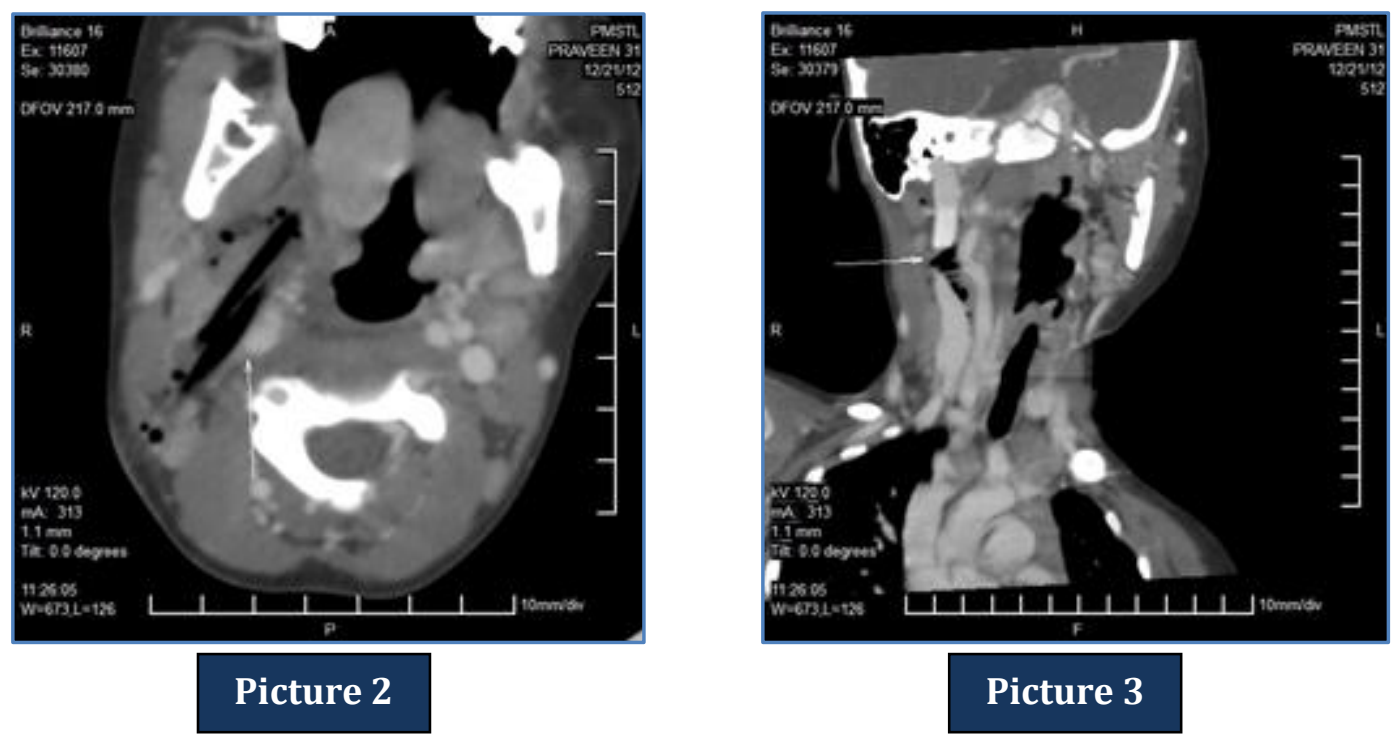

The removal of vegetative foreign body was done by transcervical approach under general anesthesia. The wooden stick (length $7 \mathrm{~cm}$ picture 5) was found between the internal jugular vein and carotid artery. The internal jugular vein was compressed and thrombosed at the level of foreign body (picture 4). The IJV was ligated transfixed and cut at its proximal and distal end. Then the foreign body was removed in toto. The postoperative period was uneventful. The patient is under follow up from one year without any symptom.

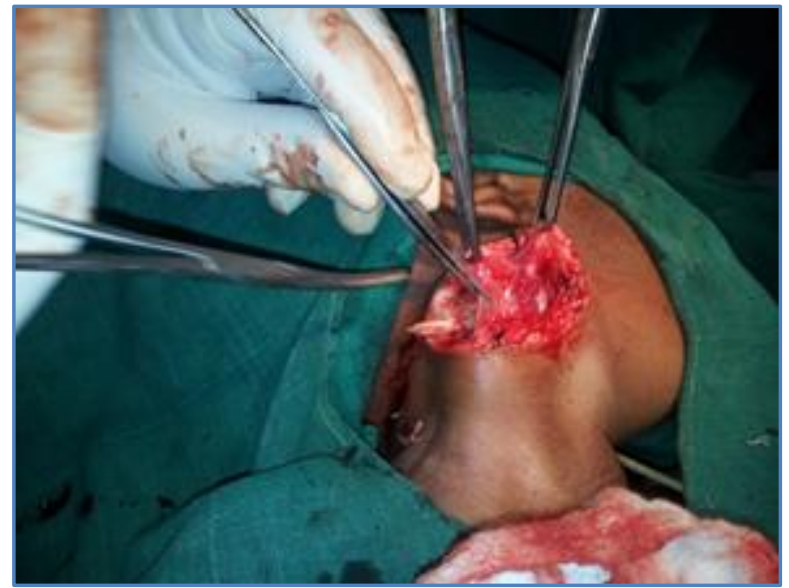

Picture 4

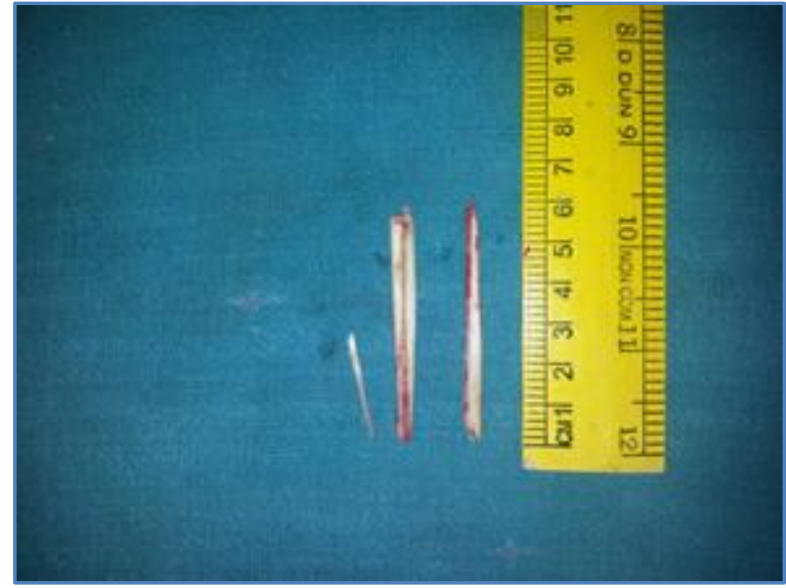

Picture 5 


\section{CASE REPORT}

DISCUSSION: Since time immemorial, foreign bodies would have been a lowbrow amusement to the clinician as well as to the population. Sometimes it may turn out uneventfully; otherwise it endangers the life of the patient depending on the type, size and location of the foreign body. The penetrating injury of the neck with a wooden stick may pose a diagnostic and therapeutic dilemma. ${ }^{3}$

Thorough knowledge of the anatomy of the neck, physical assessment and current recommendations for diagnostic and therapeutic interventions are necessary for appropriate management. The aggregation of the vital structures located in a small volume of the neck make them more vulnerable for damage in any penetrating injury of the neck and life-threatening consequences.

In most of the instances, metal, broken glass and projectile was the common object causing penetrating foreign body in the neck. ${ }^{4}$ It is skeptical to lodge a wooden in the neck, piercing through the tonsillar fossa and travelling in the carotid triangles, as seen in this case. The wooden foreign bodies are dirty and infectious because the porous organic material provides good culture conditions for gram positive and gram negative bacteria, which may cause abscess formations. ${ }^{4}$

Although a significant number of studies recommended the CT scan as the investigation of choice for the stable case of penetrating neck injury ${ }^{5}$ other investigations only enlighten the clinician about the few facts of injury. Selective arterial angiography is an important tool in diagnosing vascular injury in the case of penetrating neck injury. ${ }^{6}$

The treatment modality for vegetative foreign body is early exploration as we did in our case. Postoperative intensive broad-spectrum antibiotic treatment should be administered to prevent late infectious events. Delay in surgical intervention and improper debridement may lead to abscess formation, tissue necrosis, fibrosis and wound contracture.

CONCLUSION: The present case is interesting because of the mode of entry of the foreign body, the way it travels through tonsillar fossa to carotid triangle of the neck, between the IJV and carotid artery.

\section{REFERENCES:}

1. Neet Hariharan. Penetrating injury neck - an unusual presentation. Indian J Otolaryngol Head Neck Surgery 2004; vol 56: 237-239

2. B Vishwanatha, A Sagayaraj, Shalini G Huddar, Prashanth Kumar, RK Datta. Penetrating neck injuries. Indian J Otolaryngol Head Neck Surgery 2007; 59: 221-224.

3. Rajnish Chander Sharma, Sunder Singh Dogra, Vikram K Mahajan. Oro - pharyngo - laryngeal foreign bodies. Indian J Otolaryngol Head neck Surgery 2012; 64(2): 197-200.

4. Anne Vikram, Apoorva Mowar, Sanjeev Kumar. Wooden foreign body Embedded in the Zygomatic Region for 2 Years. J Maxillofacial Oral surgery 2012;11(1):96-100

5. Curtis Offiah, Edward Hall. Imaging assessment of penetrating injury of the neck and face. Insights imaging 2012;3:419-431

6. Jason Pasley, Regan J Berg, Kenji Inaba. MDCTA for penetrating neck injuries. Rambam maimonidies medical journal volume 2012;3:3 


\section{CASE REPORT}

\section{AUTHORS:}

1. Raman Choudhary

2. H.S. Bhuie

3. Navneet P. Mathur

4. Arjun Nath Yogi

5. Hitesh Verma

\section{PARTICULARS OF CONTRIBUTORS:}

1. Senior Resident, Department of ENT, R.N.T Medical College, Udaipur, Rajasthan.

2. Professor and HOD, ENT, R.N.T Medical College, Udaipur, Rajasthan.

3. Assistant Professor, ENT, R.N.T Medical College, Udaipur, Rajasthan.

4. Junior Resident, ENT, R.N.T Medical College, Udaipur, Rajasthan.
5. Assistant Professor, ENT, R.N.T Medical College, Udaipur, Rajasthan.

\section{NAME ADDRESS EMAIL ID OF THE CORRESPONDING AUTHOR:}

Dr. Raman Choudhary,

Senior Resident,

Department of ENT,

R. N. T. Medical College,

Udaipur-313001, Rajasthan.

Email: raman.choudhary56@yahoo.com

Date of Submission: 10/05/2014.

Date of Peer Review: 11/05/2014.

Date of Acceptance: 17/05/2014.

Date of Publishing: 24/05/2014. 